\title{
Research Article \\ eIF4E Overexpression Is Associated with Poor Prognoses of Ovarian Cancer
}

\author{
Jun Zheng, ${ }^{1}$ Xueqing Li, ${ }^{1}$ Chunyan Zhang, ${ }^{2}$ and Yiqiang Zhang $\mathbb{D}^{1}$ \\ ${ }^{1}$ Department of Biochemistry, Changzhi Medical College, Changzhi, Shanxi, China \\ ${ }^{2}$ Department of Gynaecology and Obstetrics, Heping Hospital Affiliated to Changzhi Medical College, Changzhi, Shanxi, China
}

Correspondence should be addressed to Yiqiang Zhang; yiqiangzhang@czmc.edu.cn

Received 30 July 2020; Revised 20 September 2020; Accepted 30 November 2020; Published 12 December 2020

Academic Editor: Lubna H. Tahtamouni

Copyright (c) 2020 Jun Zheng et al. This is an open access article distributed under the Creative Commons Attribution License, which permits unrestricted use, distribution, and reproduction in any medium, provided the original work is properly cited.

\begin{abstract}
Aim. Ovarian cancer is a common malignant tumor of the gynecological oncology worldwide, with a high incidence and mortality rate and poor prognosis. Searching for new diagnostic molecular biomarkers for ovarian cancer is extremely significant. Methods. Here, we analyzed the expression rates of eIF4E and cyclin D1 proteins in 123 cases of cancer tissue samples and 38 cases of paracancerous tissue samples and studied the connection between the expression rates of eIF4E and cyclin D1 proteins by immunohistochemistry and statistically correlated with clinicopathological features in ovarian cancer. Results. The results showed that the expression rates of eIF4E and cyclin D1 proteins in ovarian cancer tissues were significantly higher than those in noncancerous epithelial ovarian tissues $(P=0.001$ and $P=0.032$, respectively). Additionally, the results revealed that a higher expression rate of eIF4E $(P=0.008)$ was found in the advanced stage (stage III/IV), and also patients with cervical lymph node metastasis displayed higher expression of eIF4E $(P<0.001)$ and cyclin D1 $(P=0.033)$ than those without lymph node metastasis. Spearman's rank correlation test showed that there was a significant positive correlation between the eIF4E and cyclin D1 proteins in ovarian cancer. The Kaplan-Meier method showed that patients with lower expression of eIF4E had marginally better survival than those with high expression of eIF4E $(P=0.012)$. Multivariate Cox regression analysis further identified that positive expression of eIF4E was an independent prognostic factor. Conclusion. In ovarian cancer, eIF4E might be a valuable biomarker to predict poor prognoses and a potential therapeutic target to develop valid treatment strategies.
\end{abstract}

\section{Introduction}

Ovarian cancer is a common type of gynecological cancer worldwide, with a high incidence and mortality rate. Pieces of evidence indicate that ovarian cancer remains the fifth cause of cancer-related death among women in the United States. Over the last three decades, the five-year survival rate of ovarian cancer patients has remained at a low level with $35-46 \%[1,2]$. The high mortality rate and poor prognosis of ovarian cancer patients are due to the lack of early symptoms and early effective screening tests. So, most patients are first diagnosed at the advanced stage or metastasis. In addition, although traditional therapy such as platinumbased treatment has been discovered more than 30 years ago, the overall survival rate of women has changed little now because of the advanced resistance. Although these patients have been treated with first-line surgery and chemo- therapy, most of them will still face relapse and death $[3,4]$. So far, there are no reliable molecular markers to predict aggressive phenotypes. Therefore, searching for new molecular biomarkers for ovarian cancer is extremely urgent, which may provide a potential screening test for early detection and reveal a novel therapeutic strategy to improve the survival of ovarian cancer patients.

Protein translational regulation is an important strategy to control gene expression in normal eukaryotic cells. Abnormal expression of specific proteins such as c-MYC and cyclin D1 can lead to tumorigenesis $[5,6]$. The main target of the translational regulation factor is eukaryotic translation initiation factor 4E (eIF4E), which has been considered a limiting factor in the regulation of gene expression and affects many essential cellular processes in eukaryotic cells because it is the least abundant initiator factor involved in the eIF4F complex $[7,8]$. The activity of eIF4E is regulated by multiple 
aspects. The $4 \mathrm{EBP} 1$ profilin of eIF4E prevents the assembly of eIF4F by competing with eIF4G to bind to eIF4E. When the 4EBP1 protein is phosphorylated by the AKT/mTOR signal pathway, eIF4E will be free from $4 \mathrm{EBP} 1$ to bind with eIF4G and then form the $4 \mathrm{~F}$ complex, which subsequently promotes the translation of specific proteins [9] (shown in Figure 1). Overexpression of eIF4E is the main mechanism for eIF4E activation and has been discovered in many cancer tissues. Under physiological conditions, eIF4E with a low expression level can play a normal role in cells. When eIF4E is overexpressed, it can selectively affect the translation rate of some certain proteins, thus inducing cell proliferation and promoting the antiapoptosis, invasion, recurrence, and metastasis of tumor cells. Previous studies have confirmed that eIF4E was overexpressed in multiple tumor tissues including breast, lung, head, and neck tumors [9-13]. Hence, overexpression of eIF4E might be a biological marker for malignant tumors, with clinical significance of poor prognoses. We believed that changes in the eIF4E level might affect the translation rate of certain proteins, particularly those related to cell growth and survival involved in oncogenesis, invasion, and metastasis. Some studies have shown that eIF4E was correlated with the occurrence and development of human cancers, and the inhibition of eIF4E expression acted as a potential therapeutic target [13-18]. Previous studies have substantiated that the expression of eIF4E protein was closely related to clinicopathologic features and poor prognoses [19]. However, whether overexpression of eIF4E is associated with the development and progression of ovarian cancer has not been reported. In the current study, we analyzed the expression of eIF4E and cyclin D1 proteins in 123 ovarian cancer specimens and 38 noncancerous epithelial ovarian specimens by immunohistochemistry (IHC) and investigated the correlation between the expression of these two proteins and clinicopathologic/prognostic characteristics in ovarian cancer.

\section{Materials and Methods}

2.1. Tissue Samples and Clinical Data. One hundred and twenty-three (123) cases of paraffin-embedded ovarian cancer tissues and thirty-eight (38) cases of paracancerous tissues were collected from the Peace Hospital Affiliated to Changzhi Medical College (Changzhi, China). The patients' age was between 35 and 73 years (median, 51.8 years). All specimens and protocols were approved by the Ethics Review Board with informed consent. All patients had not received chemotherapy/radiotherapy before the biopsy. All samples were fixed with $4 \%$ neutral formalin and embedded with paraffin. Clinical data including age, clinical stages, lymph node metastasis, and survival status were available for each patient as shown in Table 1.

2.2. IHC and Scores. The IHC staining for eIF4E and cyclin D1 proteins in ovarian cancer samples was carried out with ready-to-use EnVision ${ }^{\mathrm{TM}}+$ Dual Link System-HRP methods (Dako, USA). Briefly, each section was regularly dewaxed through xylene followed by rehydration with a gradient of alcohols; subsequently, high-temperature antigen retrieval was achieved by heating the sections for $15 \mathrm{~min}$ in a $10 \mathrm{mM}$ citrate buffer ( $\mathrm{pH}$ 6.0) in a microwave, and then the sections were immersed into $3 \%$ hydrogen peroxide $\left(\mathrm{H}_{2} \mathrm{O}_{2}\right)$ in methanol for $15 \mathrm{~min}$. Sections were incubated with $5 \%$ normal goat serum for $30 \mathrm{~min}$ at room temperature. After that, the sections were incubated with a primary antibody, including eIF4E (pS473) protein (Epitomics, Inc., USA) in $1: 200$ dilution and cyclin D1 protein (Cell Signaling, USA) in $1: 200$ dilution at $4^{\circ} \mathrm{C}$ overnight, and washed thoroughly with PBS, and a secondary antibody (Maixin Biotechnology Inc., China) conjugated with a labeled polymer-HRP was incubated at room temperature for 50 minutes. The color reaction was developed by using DAB (Maixin Biotechnology Inc., China). Positive and negative controls were included in every experiment.

Immunohistochemical staining was estimated independently by two pathologists. The eIF4E and cyclin D1 staining was scored as negative ( $<10 \%$ staining) and positive $(>10 \%$ staining), respectively [19].

2.3. Statistical Analyses. The chi-squared test was used to evaluate the relationship between the expression of eIF4E and cyclin D1 and clinicopathological characteristics. The Kaplan-Meier method was used to calculate the survival curves. Spearman's rank correlation coefficient was performed to assess the relationship between the eIF4E and cyclin D1 proteins. The Cox proportional hazard regression model was used to evaluate whether the expression rates of eIF4E and cyclin D1 proteins were independent prognostic factors. For all analyses, $P$ values were two-sided and $P<$ 0.05 was considered statistically significant. All statistical analyses were performed using SPSS 18.0 (Statistical Package for the Social Sciences, Version 18.0).

\section{Results}

3.1. eIF4E and Cyclin D1 Proteins Were Overexpressed in Ovarian Cancer Tissues. We examined the cellular location and expression level of eIF4E and cyclin D1 proteins in ovarian cancer tissues by IHC. Strong positive staining of eIF4E was found in the cytoplasm and nucleus of ovarian cancer tissues (Figure 2). And positive staining of cyclin D1 was identified in the cell cytoplasm of ovarian cancer tissues. As shown in Table 2, the positive expression rate of eIF4E and cyclin D1 in ovarian cancer tissues was 68.3\% (84/123) and $64.2 \%$ (79/123), respectively. And in noncancerous epithelial ovarian tissues, the expression rate was 39.5\% (15/38) and $44.7 \%$ (17/38), respectively. The expression rate of eIF4E and cyclin D1 in ovarian cancer tissues was significantly higher than that in noncancerous epithelial ovarian tissues ( $P=0.001$ and $P=0.032$, respectively).

3.2. eIF4E and Cyclin D1 Proteins Were Related to Clinicopathological Features of Ovarian Cancer. We further studied the correlation between the expression of eIF4E and cyclin D1 proteins and clinicopathological features of ovarian cancer, including age, clinical stage, lymph node metastasis status, and survival status by a univariate chi-squared test (Table 1). Statistical analyses revealed that higher positive 


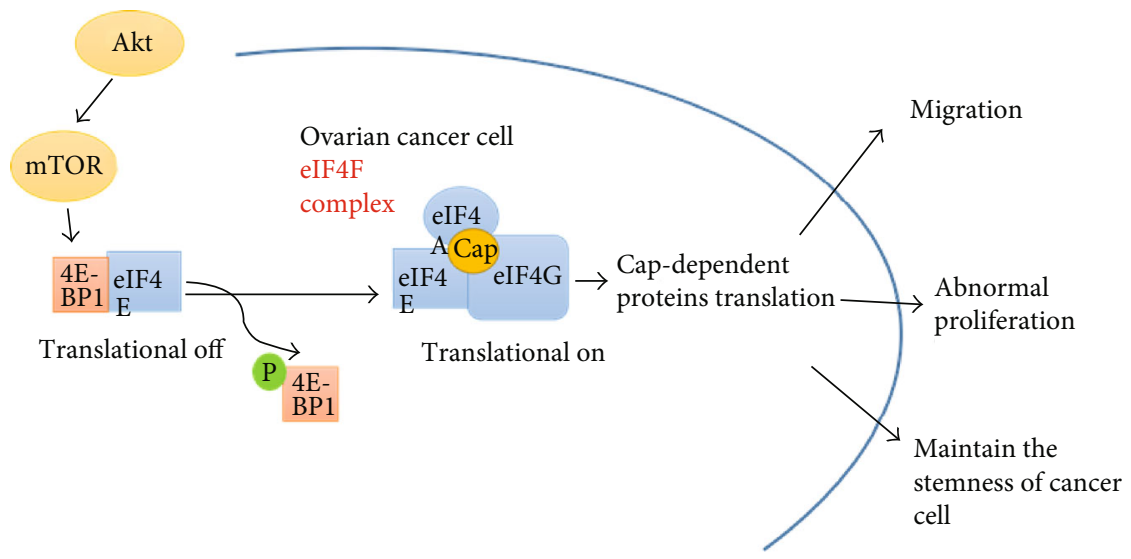

FIGURE 1: When the 4EBP1 protein is phosphorylated by the AKT/mTOR signal pathway, eIF4E will be free from 4EBP1 to bind with eIF4G and then form the eIF4F complex, which subsequently promotes the translation of cap-dependent proteins. These special proteins could promote migration and abnormal proliferation and maintain the stemness of ovarian cancer cells.

TABle 1: Association between the expression of eIF4E and cyclin D1 proteins and clinicopathological features of ovarian cancer patients.

\begin{tabular}{|c|c|c|c|c|c|c|c|c|c|}
\hline \multirow{2}{*}{ Characteristics } & \multicolumn{3}{|c|}{ eIF4E } & \multicolumn{3}{|c|}{ Cyclin D1 } & \multicolumn{3}{|c|}{ P-4EBP1 } \\
\hline & $+(\%)$ & - (\%) & $P$ & $+(\%)$ & - $(\%)$ & $P$ & $+(\%)$ & - (\%) & $P$ \\
\hline \multicolumn{10}{|l|}{ Age (yr) } \\
\hline$\leq 55(n=48)$ & $32(66.7)$ & $16(33.3)$ & 0.096 & $30(62.5)$ & $18(37.5)$ & 0.102 & $26(54.2)$ & $22(45.8)$ & 0.008 \\
\hline$>55(n=75)$ & $52(69.3)$ & $23(30.7)$ & 0.757 & $49(65.3)$ & $26(34.7)$ & 0.749 & $40(53.3)$ & $35(46.7)$ & 0.928 \\
\hline \multicolumn{10}{|l|}{ Clinical stages } \\
\hline I-II $(n=37)$ & $19(51.4)$ & $18(48.6)$ & 7.014 & $25(67.6)$ & $12(32.4)$ & 0.257 & $17(45.9)$ & $20(50.1)$ & 1.266 \\
\hline III-IV $(n=86)$ & $65(75.6)$ & $21(24.4)$ & 0.008 & $54(62.8)$ & $32(37.2)$ & 0.612 & $49(57.0)$ & $37(43.0)$ & 0.261 \\
\hline \multicolumn{10}{|l|}{ LN status } \\
\hline $\operatorname{LNM}(n=82)$ & $68(82.9)$ & $14(17.1)$ & 24.330 & $58(70.7)$ & $24(29.3)$ & 4.519 & $53(64.6)$ & $29(35.4)$ & 11.917 \\
\hline No LNM $(n=41)$ & $16(39.0)$ & $25(61.0)$ & $<0.001$ & $21(51.2)$ & $20(48.8)$ & 0.033 & $13(31.7)$ & $28(68.3)$ & 0.001 \\
\hline \multicolumn{10}{|l|}{ Survival status } \\
\hline Dead $(n=88)$ & $68(77.3)$ & $20(22.7)$ & 11.517 & $59(67.0)$ & $29(33.0)$ & 1.069 & $55(62.5)$ & $33(37.5)$ & \\
\hline Alive $(n=35)$ & $16(45.7)$ & $19(54.3)$ & 0.001 & $20(57.1)$ & $15(42.9)$ & 0.301 & $11(31.4)$ & $24(68.6)$ & 0.002 \\
\hline
\end{tabular}

Abbreviations: LN: lymph node; LNM: lymph node metastasis. A chi-squared test was used to evaluate the relationship between the expression of eIF4E and cyclin D1 and clinicopathological characteristics.

expression of eIF4E $(P=0.008)$ was found in the advanced (III/IV) stage than the early (I/II) stage, and patients with cervical lymph node metastasis had higher expression of eIF4E $(P<0.001)$ and cyclin D1 $(P=0.033)$ than those without lymph node metastasis. The results also showed that strong positive expression of eIF4E was related to the survival status of ovarian cancer patients $(P=0.001)$. However, the expression of cyclin D1 had no significant differences with the clinical stage (I/II or III/IV) and survival status $(P>0.05$, respectively). Moreover, the positive expression of eIF4E and cyclin D1 proteins was not related to the age of ovarian cancer patients $(P>0.05$, respectively).

\section{3. eIF4E Had a Positive Correlation with Cyclin D1 Proteins} in Ovarian Cancer. We further analyzed the correlation between the expression rates of eIF4E and cyclin D1 proteins in ovarian cancer using Spearman's rank correlation test (Table 3). Data showed that there was a strong positive cor- relation between the eIF4E and cyclin D1 proteins $(r=2.453, P=0.002)$.

3.4. Overexpression of eIF4E Protein Was Associated with Poor Prognoses. We obtained the follow-up data of all patients and studied the association between the expression of eIF4E and cyclin D1 proteins and overall survival using the Kaplan-Meier method. Figure 3 displays some evidence of survival advantage for ovarian cancer patients with negative expression of eIF4E and cyclin D1. The ovarian cancer patients with lower expression of eIF4E had a better survival rate than those with strong expression of eIF4E $(P=0.012$, Figure 3(a)). Although patients had better survival outcomes with negative expression of cyclin D1, it was not statistically significant $(P>0.05$, Figure 3(b)). Meanwhile, we investigated potential correlations of independent prognostic factors including clinical stages, lymph node metastasis status, and expression of eIF4E and cyclin D1 proteins for ovarian 


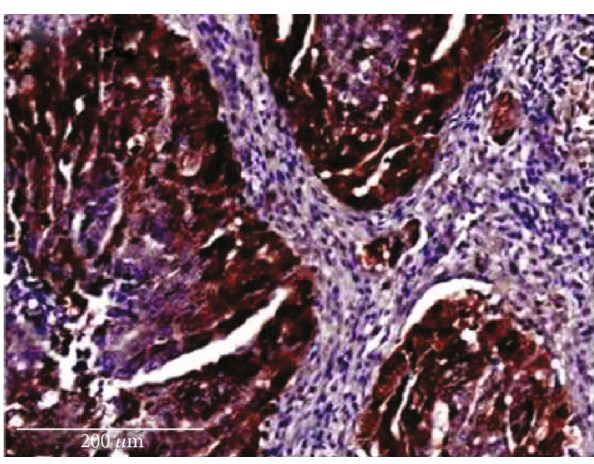

(a)

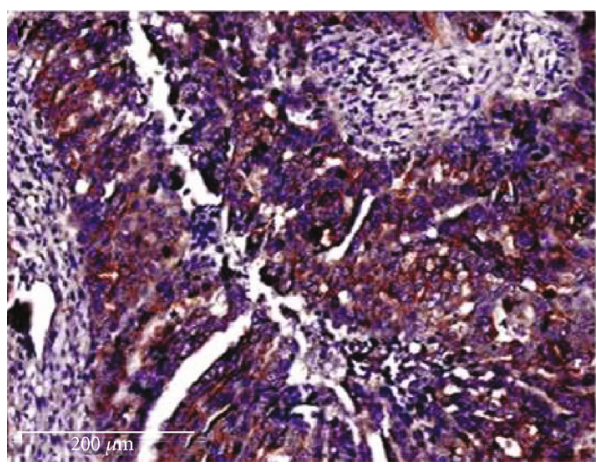

(c)

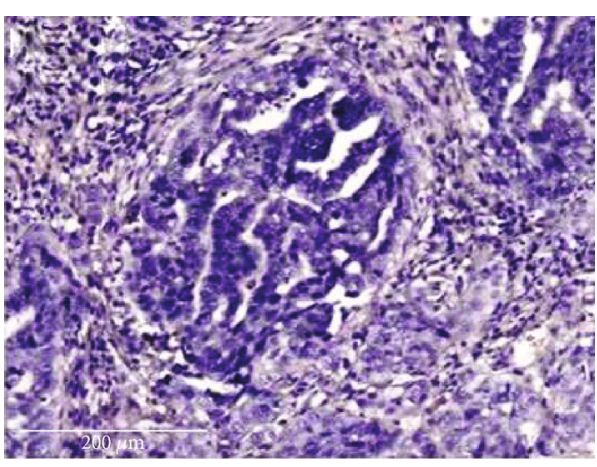

(b)

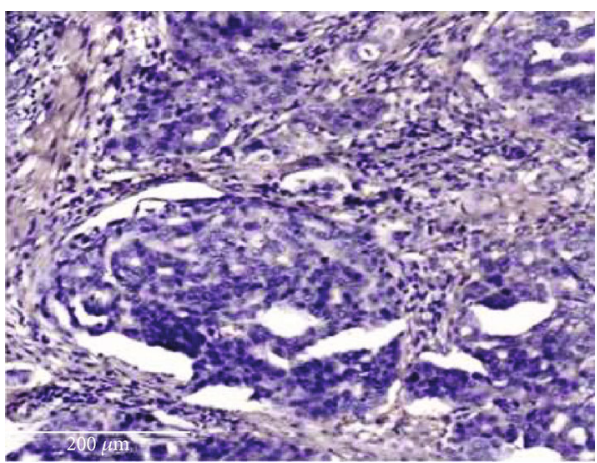

(d)

FIgURE 2: Overexpression of eIF4E and cyclin D1 proteins in ovarian cancer tissues. The expression of eIF4E and cyclin D1 proteins was detected by immunohistochemistry using specific antibodies as described in Materials and Methods. Strong positive ((a), DAB staining) and negative ((b), DAB staining) expression rates of eIF4E protein were shown in the ovarian cancer tissues. Strong positive ((c), DAB staining) and negative ((d), DAB staining) expression rates of cyclin D1 protein were shown in ovarian cancer tissues.

TABLE 2: The expression of eIF4E and cyclin D1 proteins in ovarian cancer tissues and noncancerous ovarian tissues.

\begin{tabular}{|c|c|c|c|c|}
\hline \multirow{2}{*}{ Histologic type } & \multicolumn{2}{|c|}{ eIF4E } & \multicolumn{2}{|c|}{ Cyclin D1 } \\
\hline & $+(\%)$ & - (\%) & $+(\%)$ & - (\%) \\
\hline $\mathrm{OC}(n=123)$ & $84(68.3)$ & $39(31.7)$ & $79(64.2)$ & $44(35.8)$ \\
\hline NOC (38) & $15(39.5)$ & $23(60.5)$ & $17(44.7)$ & $21(55.3)$ \\
\hline$\chi^{2}$ & \multicolumn{2}{|c|}{10.182} & \multicolumn{2}{|c|}{4.581} \\
\hline$P$ & \multicolumn{2}{|c|}{0.001} & \multicolumn{2}{|c|}{0.032} \\
\hline
\end{tabular}

Abbreviations: OC: ovarian cancer tissues; NOC: noncancerous ovarian tissues. A chi-squared test was used to detect the difference of expression of eIF4E and cyclin D1 proteins in ovarian cancer tissues and noncancerous ovarian tissues.

TABLE 3: The pairwise association between the expression of eIF4E and cyclin D1 proteins in ovarian cancer patients.

\begin{tabular}{lccc}
\hline eIF4E & Positive (\%) & $\begin{array}{c}\text { Cyclin D1 } \\
\text { Negative (\%) }\end{array}$ & $P$ value \\
\hline Positive (\%) & $66(53.7)$ & $18(14.6)$ & 0.002 \\
Negative (\%) & $13(10.6)$ & $26(21.1)$ & $(r=2.453)$ \\
\hline
\end{tabular}

Spearman's rank correlation coefficient was performed to assess the relationship between the eIF4E and cyclin D1 proteins.

cancer patients by multivariate Cox proportional hazard regression analysis. Table 4 reveals that positive expression of eIF4E protein was identified as an independent poor prognostic factor for ovarian cancer patients $(P=0.003)$, as well as clinical stages $(P=0.002)$ and lymph node metastasis sta- tus $(P=0.001)$. Nevertheless, cyclin D1 protein was not an independent poor prognostic factor $(P>0.05)$.

\section{Discussion and Conclusion}

Increasing pieces of evidence have shown that eIF4E is a speed limiting factor in the regulation of gene expression and affects many essential cellular processes, including cell growth, proliferation, cell differentiation, and survival in eukaryotic cells $[8,13,16]$. Overexpression of eIF4E has been reported in many malignant carcinomas, such as breast carcinoma, prostate cancer, nasopharyngeal carcinoma, lung carcinoma, and head and neck carcinoma, and was associated with clinicopathological features and prognostic implications [11, 14, 20-24]. Several in vitro experiments showed 


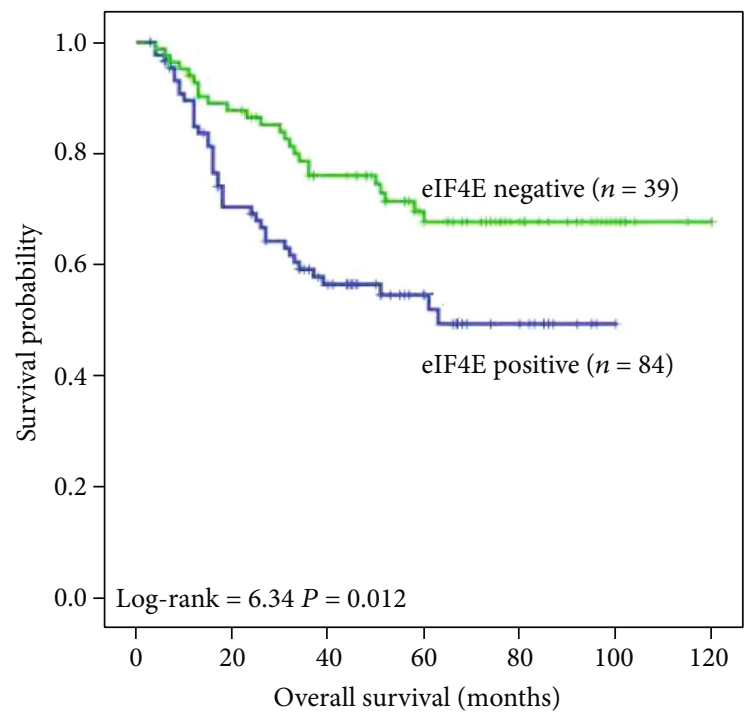

(a)

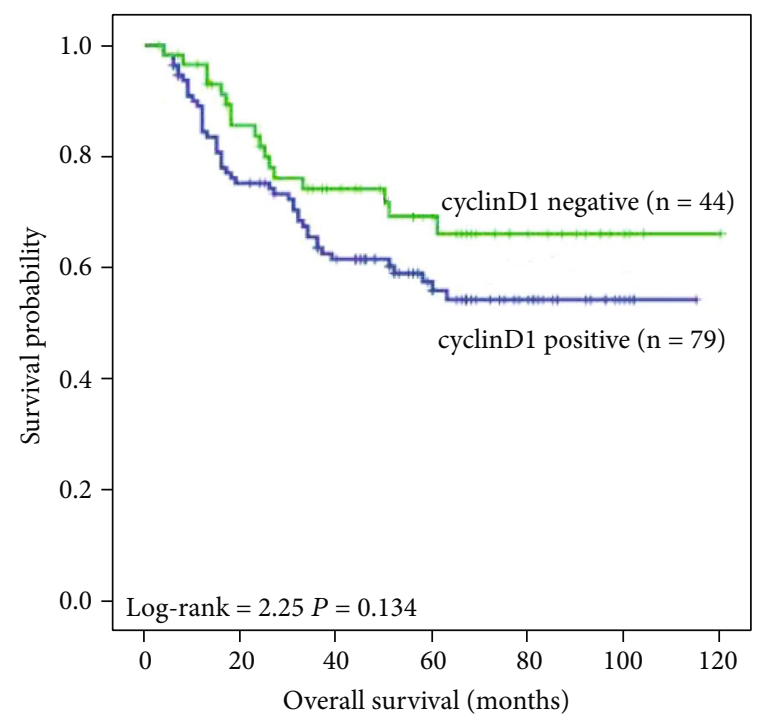

(b)

Figure 3: Kaplan-Meier overall survival curves of ovarian cancer patients. Kaplan-Meier analysis was used to plot the survival curve of all 123 ovarian cancer patients with expression of eIF4E and cyclin D1 proteins, and statistical significance was assessed using the log-rank test. (a) Kaplan-Meier curves showed that worse overall survival was seen in eIF4E-positive patients compared with eIF4E-negative patients $(P=0.012$, two-sided). (b) Kaplan-Meier curves showed that the expression of cyclin D1 protein had no significant correlation with overall survival rates of ovarian cancer patients $(P>0.05$, two-sided $)$.

TABLE 4: Summary of multivariate analysis of the Cox proportional hazard model for overall survival of ovarian cancer patients.

\begin{tabular}{|c|c|c|c|c|c|}
\hline & SE & Wald & $P$ value & $\operatorname{Exp}(B)$ & $95 \% \mathrm{CI}$ \\
\hline eIF4E expression & 0.274 & 8.785 & $0.003^{*}$ & 0.444 & $0.260-0.760$ \\
\hline Cyclin D1 expression & 0.507 & 0.130 & 0.719 & 1.200 & $0.445-3.240$ \\
\hline LN status (LNM vs. no LNM) & 0.384 & 10.561 & $0.001^{*}$ & 3.480 & $1.640-7.383$ \\
\hline Clinical stages (I-II vs. III-IV) & 0.325 & 9.201 & $0.002^{*}$ & 4.403 & $2.194-8.836$ \\
\hline
\end{tabular}

Abbreviations: LN: lymph node; LNM: lymph node metastasis. The Cox proportional hazard regression model was used to evaluate whether the expression rates of eIF4E and cyclin D1 proteins were independent prognostic factors.

that a high level of eIF4E was associated with cell proliferation and cancer progression and significantly reduced the level of antiapoptotic proteins which induced apoptosis and sensitized the cells to chemotherapy [25]. Previous studies have demonstrated that the knockdown of eIF4E led to the prolongation of G1 phase transition and G0/G1 cell cycle arrest by decreasing the translation of c-MYC and cyclin D1 in some carcinoma cell lines [26, 27]. A similar effect was observed in in vivo experiment where antisense oligonucleotide (ASO) selectively reduced the eIF4E level in human tumor xenografts and dramatically decreased tumor growth without eliciting cytotoxicity in normal tissues [28, 29]. In the previous study, the results showed that there was high positive expression of eIF4E in nasopharyngeal carcinoma tissues and it was closely related to clinicopathologic features and poor prognoses [19]. In this study, the results showed that there was higher positive expression of eIF4E and cyclin D1 in ovarian cancer than in noncancerous epithelial ovarian tissues, and eIF4E had a strong positive correlation with cyclin D1 protein. Cyclin D1 is putatively one of the weak mRNA oncogenes, and its expression is effectively increased by eIF4E overexpression $[30,31]$. The result of eIF4E overex- pression in ovarian cancer was consistent with other reports. This finding strongly indicated that eIF4E might play a crucial role in the carcinogenesis of ovarian cancer.

Tumor progression is related to recurrence and metastasis and also affects patients' prognoses. Recently, a study found that the eIF4E could enhance MMP9 expression to promote metastasis in mouse breast cancer [32]. Other studies indicated that the treatment with 4EGI1 (a competitive eIF4E/eIF4G interaction inhibitor) might affect the expression of both eIF4E and cyclin D1 in the cellular level of NPC [32]. However, whether eIF4E could promote metastasis in ovarian cancer tissues is still unknown. In our study, results showed that higher expression of eIF4E protein was found in patients with the advanced (III/IV) stage and cervical lymph node metastasis. The expression of eIF4E was negatively correlated with the survival status of patients. These results indicated that the expression of eIF4E is important in the progression of ovarian cancer. We found that the expression of cyclin D1 was higher in patients with cervical lymph node metastasis than those without lymph node metastasis, while it had no significant difference in the clinical stage (I/II or III/IV) and survival. 
There are many factors related to prognoses such as lymph node metastasis and clinical stage. eIF4E protein overexpression has been observed in many solid tumors and was correlated with recurrence, metastasis, and poor prognoses of human tumors [11, 14, 20-24]. Our results showed that patients with lower expression of eIF4E had better survival than those with strong expression. Meanwhile, multivariate analysis proved that eIF4E positive expression was an independent factor of ovarian cancer. Therefore, eIF4E may act as a novel prognostic molecular marker and be a potential molecular target for the therapy and prevention of ovarian cancer.

In summary, our results proved that the expression of eIF4E protein was higher in ovarian cancer tissues and might promote the proliferation and cell cycle progression by enhancing the translation of cyclin D1. Overexpression of eIF4E was associated with clinicopathological features and prognoses of ovarian cancer patients and might be a novel valuable biomarker to predict poor prognoses and a therapeutic target to develop valid treatment strategies. In vitro studies can improve the understanding of the molecular mechanisms involved. It is of great value and urgency to further study the relevant aspects. In ovarian cancer, eIF4E might be a valuable biomarker to predict poor prognoses and may be a therapeutic target to develop valid treatment strategies.

\section{Data Availability}

All data generated or analyzed during this study are included in this published article.

\section{Conflicts of Interest}

The authors declare that they have no conflicts of interest.

\section{Authors' Contributions}

Jun Zheng and Xueqing Li contributed equally to this work.

\section{Acknowledgments}

The work was supported by grants from the science and technology innovation project of colleges and universities in Shanxi Province (NO. 2019L0688) and the Health commission scientific research project in Shanxi Province (NO. 2019139).

\section{References}

[1] A. Jemal, R. Siegel, E. Ward, Y. Hao, J. Xu, and M. J. Thun, "Cancer statistics, 2009," CA: a cancer journal for clinicians, vol. 59, no. 4, pp. 225-249, 2009.

[2] L. Luo, L.-Y. Dong, Q.-G. Yan et al., "Research progress in applying proteomics technology to explore early diagnosis biomarkers of breast cancer, lung cancer and ovarian cancer," Asian Pacific journal of cancer prevention, vol. 15, no. 20, pp. 8529-8538, 2014.

[3] X. Tianmin, C. Weiqin, W. Shuying, L. Yang, and C. Manhua, "Protection of ovarian function during chemotherapy for ovarian cancer," European Journal of Gynaecological Oncology, vol. 35, 2014.

[4] A. Majidi, R. Na, S. Dixon-Suen, S. J. Jordan, and P. M. Webb, "Common medications and survival in women with ovarian cancer: a systematic review and meta-analysis," Gynecologic Oncology, vol. 157, no. 3, 2020.

[5] K. Tsuda-Sakurai and M. Miura, "The hidden nature of protein translational control by diphthamide - the secrets under the leather," Journal of Biochemistry, vol. 165, no. 1, pp. 1-8, 2018.

[6] T. Khoury, S. Alrawi, N. Ramnath et al., "Eukaryotic initiation factor-4E and cyclin D1 expression associated with patient survival in lung cancer," Clinical Lung Cancer, vol. 10, no. 1, pp. 58-66, 2009.

[7] P. B. Bitterman and V. A. Polunovsky, "EIF4E-mediated translational control of cancer incidence," Biochimica Et Biophysica Acta Gene Regulatory Mechanisms, vol. 1849, no. 7, pp. 74-80, 2015.

[8] P. M. Fischer, "Cap in hand: targeting eIF4E," Cell cycle, vol. 8, no. 16, pp. 2535-2541, 2009.

[9] L. Beretta, A. C. Gingras, Y. V. Svitkin, M. N. Hall, and N. Sonenberg, "Rapamycin blocks the phosphorylation of 4E-BP1 and inhibits cap-dependent initiation of translation," Embo Journal, vol. 15, no. 3, pp. 658-664, 1996.

[10] S. Diab, M. Kumarasiri, M. Yu et al., "MAP kinase-interacting kinases-emerging targets against cancer," Chemistry \& biology, vol. 21, no. 4, 2014.

[11] B. Zhang, Z. H. Chengchu, C. H. Baofu, X. Zhang, Y. E. Minhua, and L. I. Aifen, "Expression and its clinical significance of eIF4E in non-small cell lung cancer," Chinese Journal of Lung Cancer, vol. 13, no. 12, pp. 1132-1135, 2010.

[12] A. Piserà, A. Campo, and S. Campo, "Structure and functions of the translation initiation factor eIF4E and its role in cancer development and treatment," Journal of Genetics Genomics, vol. 45, no. 1, pp. 13-24, 2018.

[13] S. G. Zimmer, D. B. AU, and J. R. Graff, "Translational control of malignancy: the mRNA cap-binding protein, eIF-4E, as a central regulator of tumor formation, growth, invasion and metastasis," Anticancer Research, vol. 20, pp. 1343-1351, 2000.

[14] M. Wu, Y. Liu, X. Di et al., "EIF4E over-expresses and enhances cell proliferation and cell cycle progression in nasopharyngeal carcinoma," Medical Oncology, vol. 30, no. 1, p. 400, 2013.

[15] C. Lu, L. Makala, D. Wu, and Y. Cai, "Targeting translation: eIF4E as an emerging anticancer drug target," Expert Reviews in Molecular Medicine, vol. 18, article e2, 2016.

[16] A. De Benedetti and J. R. Graff, "eIF-4E expression and its role in malignancies and metastases," Oncogene, vol. 23, no. 18, pp. 3189-3199, 2004.

[17] R. L. A. Silva and H. G. Wendel, "MNK, EIF4E and targeting translation for therapy," Cell Cycle, vol. 7, no. 5, pp. 553-555, 2014.

[18] J. D. Richter and N. Sonenberg, "Regulation of cap-dependent translation by eIF4E inhibitory proteins," Nature, vol. 433, no. 7025, pp. 477-480, 2005.

[19] J. Zheng, J. Li, L. Xu et al., "Phosphorylated Mnk1 and eIF4E are associated with lymph node metastasis and poor prognosis of nasopharyngeal carcinoma," Plos One, vol. 9, no. 2, article e89220, 2014.

[20] T. P. Farias, F. L. Dias, R. A. Lima et al., "Prognostic factors and outcome for nasopharyngeal carcinoma," Archives of 
Otolaryngology - Head \& Neck Surgery, vol. 129, no. 7, pp. 794-799, 2003.

[21] J. R. Graff, B. W. Konicek, R. L. Lynch et al., “eIF4E activation is commonly elevated in advanced human prostate cancers and significantly related to reduced patient survival," Cancer Research, vol. 69, no. 9, pp. 3866-3873, 2009.

[22] T. Heikkinen, T. Korpela, R. Fagerholm et al., "Eukaryotic translation initiation factor 4E (eIF4E) expression is associated with breast cancer tumor phenotype and predicts survival after anthracycline chemotherapy treatment," Breast Cancer Research, vol. 141, no. 1, pp. 79-88, 2013.

[23] G. Sunavala-Dossabhoy, S. Palaniyandi, C. Clark, C. A. O. Nathan, F. W. Abreo, and G. Caldito, "Analysis of eIF4E and 4EBP1 mRNAs in head and neck cancer," Laryngoscope, vol. 121, no. 10, pp. 2136-2141, 2011.

[24] C. I. Huang, C. C. Wang, T. S. Tai et al., "eIF4E and 4EBP1 are prognostic markers of head and neck squamous cell carcinoma recurrence after definitive surgery and adjuvant radiotherapy," PLoS ONE, vol. 14, no. 11, article e0225537, 2019.

[25] G. Wang, Z. Li, Z. Li et al., "Targeting eIF4E inhibits growth, survival and angiogenesis in retinoblastoma and enhances efficacy of chemotherapy," Biomedicine Pharmacotherapy, vol. 96, pp. 750-756, 2017.

[26] J. Wan, F. Shi, Z. Xu, and M. Zhao, "Knockdown of eIF4E suppresses cell proliferation, invasion and enhances cisplatin cytotoxicity in human ovarian cancer cells," International Journal of Oncology, vol. 47, no. 6, pp. 2217-2225, 2015.

[27] B. A. Jacobson, S. C. Thumma, J. Jay-Dixon et al., "Targeting eukaryotic translation in mesothelioma cells with an eIF4Especific antisense oligonucleotide," Plos One, vol. 8, no. 11, article e81669, 2013.

[28] J. R. Graff, B. W. Konicek, T. M. Vincent et al., "Therapeutic suppression of translation initiation factor eIF4E expression reduces tumor growth without toxicity," The Journal of clinical investigation, vol. 117, 2007.

[29] A. G. Duffy, O. V. Makarova-Rusher, S. V. Ulahannan et al., "Modulation of tumor eIF4E by antisense inhibition: a phase I/II translational clinical trial of ISIS 183750-an antisense oligonucleotide against eIF4E-in combination with irinotecan in solid tumors and irinotecan-refractory colorectal cancer," International Journal of Cancer, vol. 139, no. 7, pp. 16481657, 2016.

[30] R. J. Jackson, C. U. T. Hellen, and T. V. Pestova, “The mechanism of eukaryotic translation initiation and principles of its regulation," Nature Reviews Molecular Cell Biology, vol. 11, no. 2, pp. 113-127, 2010.

[31] B. Culjkovic, I. Topisirovic, L. Skrabanek, M. Ruiz-Gutierrez, and K. L. B. Borden, "eIF4E promotes nuclear export of cyclin D1 mRNAs via an element in the 3'UTR," Journal of Cell Biology, vol. 169, no. 2, pp. 245-256, 2005.

[32] C. G. Gkogkas, A. Khoutorsky, R. Cao et al., "Pharmacogenetic inhibition of eIF4E-dependent Mmp9 mRNA translation reverses fragile X syndrome-like phenotypes," Cell Reports, vol. 9, no. 5, pp. 1742-1755, 2014. 